\title{
Soldner Had Found in 1802 the Deflection of the Light by the Sun as the General Relativity Shows
}

\author{
Marc Mignonat \\ Société d'Astronomie des Pyrénées Occidentales, Pau, France \\ Email: mmignona t@libertysurf.fr
}

How to cite this paper: Mignonat, $M$. (2018) Soldner Had Found in 1802 the Deflection of the Light by the Sun as the General Relativity Shows. Journal of Modern Physics, 9, 1545-1558.

https://doi.org/10.4236/jmp.2018.98095

Received: May 3, 2018

Accepted: July 15, 2018

Published: July 18, 2018

Copyright $\odot 2018$ by author and Scientific Research Publishing Inc.

This work is licensed under the Creative

Commons Attribution International

License (CC BY 4.0).

http://creativecommons.org/licenses/by/4.0/

\begin{abstract}
Systematically, it is written in the literature that only the general relativity (GR) allows finding the just value of the deflection of the light by the sun. Yet, we noted, by reading over the original text of SOLDNER of 1801: "Ueber die Ablenkung eines Lichtstrals von seiner geradlinigen Bewegung, durch die Attraktion eines Weltkörpers, the welchem er nahe vorbei geht" (that we think it is important to put in English in full in Appendix) that, contrary to what we read since about 100 years, he found the right value. Soldner had started from a Newtonian gravitational calculation and, with the value of 1801, find 1.64". This calculation, with the actual values, allows finding the right value of 1.752". There are reasons to explain the wrong calculations which we usually make. However, there is no epistemological reason for questioning the general relativity. Some observations are only explained by the GR. But the Newtonian calculations are much simpler. We can continue to say that the theory of Newton is incomplete but we cannot say it is false.
\end{abstract}

\section{Keywords \\ Cosmology: Theory}

\section{Commentary on the Article of Soldner [1]: (The Article of Soldner Is to Be Read in Appendix)}

The purpose of the article of Soldner is to determine, when we observe a star, what is the correction we have to make to compensate the angle of deflection of the light which is caused by the attraction of the Earth. And if this quantity is significant and must be added to this of the refraction: "However, since also the ray-refraction is a function of height, then these two quantities must be mutually 
combined..." In its article, Soldner calculates, by leaving of an inverse route Earth stars, what is the angle of deflection (" $\operatorname{tang} \omega=A B / A D$ ") and particularly what is the maximal angle of the light then horizontally arriving on the Earth. This, to correct "he aberration", exists when we observe a star. He explains that "for convenience of the study", "the light ray does not arrive at the place of observation, but emanates from it." So, it was a clever way, at that time, to eliminate the problem of the speed variation of the corpuscle. The light being considered as a corpuscle, the attraction acts and so, the speed is varied. The authors, at this time (e.g. Laplace which Soldner refers), eliminated the problem with a light ray coming from the infinite, so the variation of the speed was insignificant in the zone of observation.

Other authors had made a calculation about the deflection of the light with the Newtonian method.

Michell in 1784 [2] starts from the study of the double stars and makes an analysis with the geometrical method of Newton. He suggests that corpuscles of light are attracted by gravitational forces. He specifies for which conditions the light cannot go out of the star and so, introduces the concept of the black hole.

Laplace after replacing the geometrical method of Newton by our modern mathematical analysis, in 1796 [3], from the escape velocity, calculated the exact radius (the future radius of Schwarzschild) where the light cannot go out from "un corps obscur" (a dark body).

Will in 1988 [4] wrote that: The gravitational deflection of light based on Newtonian theory and the corpuscular model of light was calculated, but never published, around 1784 by Henry Cavendish, almost 20 years earlier than the first published calculation by Johann Georg von Soldner. The two results are slightly different because, while Cavendish treated a light ray emitted from infinity, von Soldner treated a light ray emitted from the surface of the gravitating body.

Another difficulty is because of the units used in 1800 with, e.g., the velocities measured in units of length. This induce a definition of the acceleration $g=s / t^{2}$ and $v=2 g t$ (for us, two misprints of a factor of two since $v=g t$ and $g=s / 2 t^{2}$ ), but, in the calculation, these apparent mistakes cancel at the end. So, his calculation is right.

His plan (Figure 3 in his text, Figure 1 in this letter) is particularly clear: Soldner does his calculation on a light arriving on the celestial body and not on a ray of light which only pass and continue its course. The angle $\omega$ he calculates is the one of the light coming from an infinite distance and finishing its course "in the eye of the observer" ("ins Auge des Beobachters") situated on Earth.

The light arriving on earth and deflected by the earth has a maximal deflection of $\omega=0 " .001$.

After he specifies if we take the light passing near the moon and arriving on earth, it would be necessary to double the value to take into account the 2 arms of the hyperbola... (der an dem Monde vorbey und auf die Erde geht, zwey Arme 


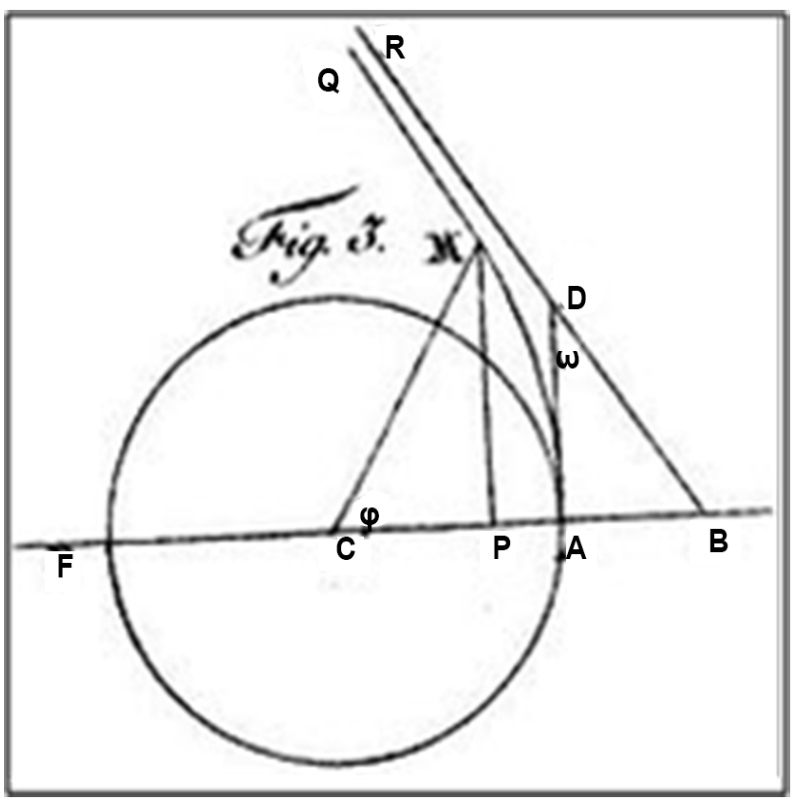

Figure 1. Original figure of Soldner where the light come from $\mathrm{Q}$ and finishes in A "in the eye of the observer".

der Hyperbel beschreibt). Also, we can note he calculates the conditions where the trajectory would be a parabola, an ellipse, a circle...

Then, he says that if the earth was replaced by the sun, the maximal deflection of the light arriving on the celestial body, the angle $\omega$ would be 0.84 (The light follows only an arm of the hyperbola).

It is the light arriving on sun and deflected by the sun and Soldner said (or jokes: "If it were possible to observe the fixed stars very nearly at the sun,... However, as it is well known that this doesn't happen...")

In this case, if we double the value of 0 " 84 , (to take into account the 2 arms of the hyperbola), Soldner finds the right value of the deflection of the light passing near the sun, which is 1"64 (with the known values of the masses, of the radius and of the speeds in 1801).

\section{The Wrong Answer We Often Find in the Literature}

In the text of Soldner, I think it is possible to make an error for two conditions:

1) If we forget the definition of the angle $\omega$ by Soldner (angle of deflection of the light arriving on a celestial body and deflected by this celestial body)

2) And if only we read in the conclusion: "....then we find $\omega=0$ ".84. If it were possible to observe the fixed stars very nearly at the sun, then we would have to take this into consideration...")

\subsection{The Pseudo Error of Newton?}

The reasoning, said of Newton Soldner, sometimes, is presented in the literature or on the net. A common mistake comes from the fact that the trajectory has been decomposed with three parts (Figure 2). It is considered the first and the 


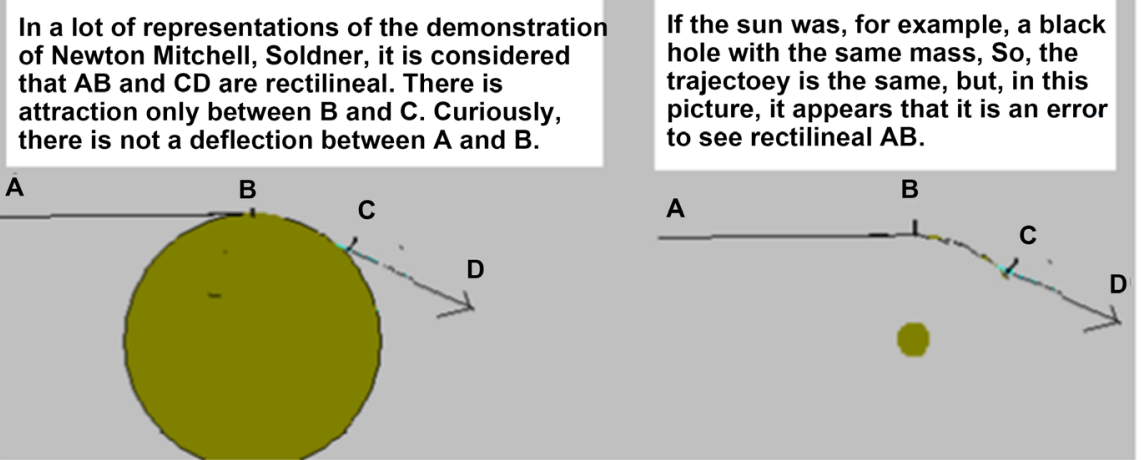

Figure 2. A common error in the representation of the trajectory of the light.

third parts are rectilineal. In the first part, the trajectory is rectilineal between infinite and the tangential point to the solar surface. So, it is considered the attraction is not exercised when the photon comes near to the Sun; the attraction is only exercised when the photon goes away! So, it is logical to find only the exact half of the correct value. Recently, in 2017, Huang [5], had also found the mistake and had made a correct calculation with a Newtonian method and clear figures. He says that there are only "minor errors" but without saying which ones, in the calculation of Soldner.

A second common error is more difficult to see (Figure 3) and, sometimes, students are provided with this figure. The deviation is also after the point of the trajectory which is tangential to the sun, but the tangential point is in A. Yet, the deviation is the same before and after a tangential point to the surface of the sun. In the figure, there is no tangential point in the zone of deviation. To have a tangential point we have to move the sun in $\mathrm{A}$ or turn the sun to have a radius perpendicular to the trajectory, i.e. we move the point $\mathrm{A}$ to this new point. We find again the problem of the Figure 2.

\subsection{We Redid in Appendix 2 the Calculation of Soldner by a More Modern Method}

The calculation was made or from the speeds or from the work of the forces with simplifications and so, perhaps the same objections identified by Soldner in his conclusion. If we take a figure with the correct path (Figure 4), the integral is made over the entire trajectory and not just half of it. The calculation allows finding the true value of the deflection of the light, so 1.75 ”.

\subsection{Hypotheses and Conclusion}

It would be possible to say that the gravitational theory of Newton and the general relativity must not be so much put in contradiction. The general relativity is a more "beautiful" theory, an explanatorier theory for the curvatures, for the absence of center...

The advance of perihelion of mercury, with the pre-1920 astronomy, is only explicable with the relativity. Perhaps, the theory of Newton is incomplete but we cannot say it is false. 


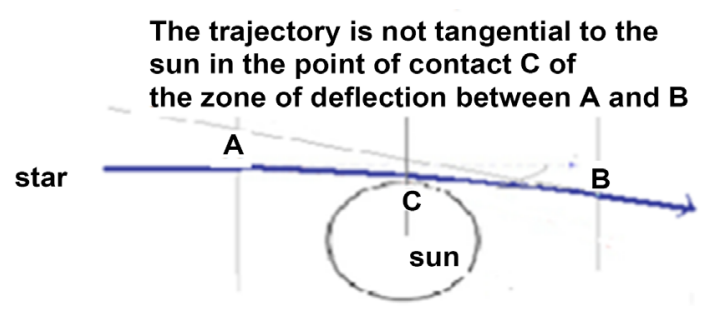

Figure 3. A second common error more difficult to see.

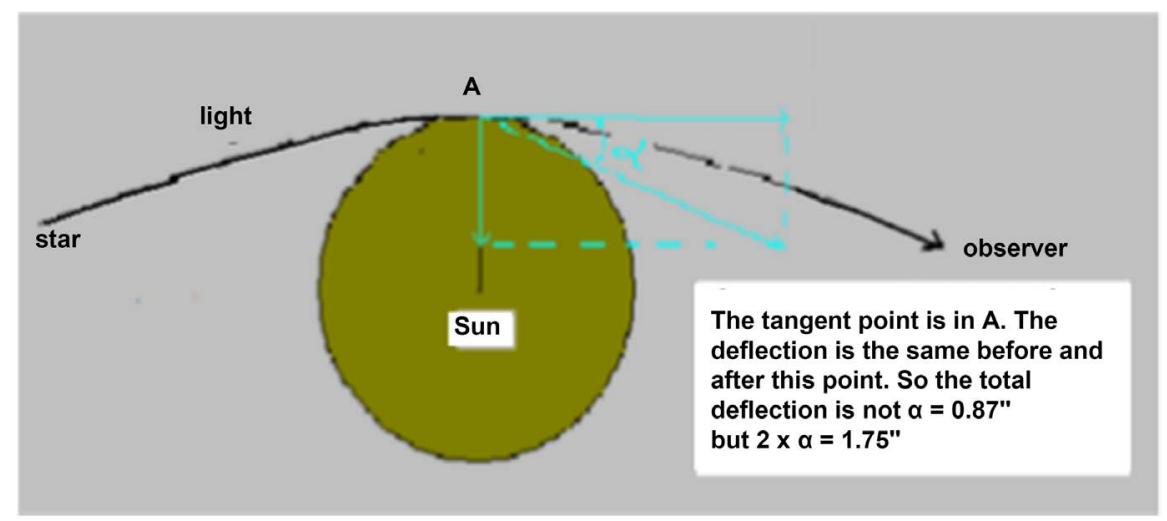

Figure 4. A representation of the path of the light with a deflection over the entire trajectory.

From a philosophic point of view, the general relativity, because coming from principles (Maupertuis, Mach) is a principle of which we can verify it is always true, and not a law in opposition with the gravitation of Newton. So, general relativity and theory of Newton perhaps are two sides or two principles of a same phenomenon.

\section{References}

[1] Soldner, J. (1804) Ueber die Ablenkung eines Lichtstrals von seiner geradlinigen Bewegung, durch die Attraktion eines Weltkörpers, an welchem er nahe vorbei geht. Berliner Astronomisches Jahrbuch, 161-172.

[2] Michell, J. (1784) Philosophical Transactions of the Royal Society, 74, 35-57. https://doi.org/10.1098/rstl.1784.0008

[3] Laplace, P.S. (1796) Mécanique céleste, livre cinquième, chap. VI: Considérations sur le système du monde et sur les progrès futur de l'astronomie, 293-312.

[4] Will, C.M. (1988) American Journal of Physics, 56, 413-415. https://doi.org/10.1119/1.15622

[5] Huang, F.Y. (2017) Journal of Modern Physics, 8, 1894-1900. https://doi.org/10.4236/jmp.2017.811112 


\section{Appendix 1}

Source:

$\underline{\text { http://en.wikisource.org/wiki/Page:Ueber_die_Ablenkung_eines_Lichtstrals_vo }}$

n_seiner_geradlinigen_Bewegung.djvu/1

On the deflection of a light ray from its rectilinear motion, by the attraction of a celestial body at which it nearly passes by.

By Joh. Soldner.

Berlin, March 1801.

At the current, so much perfected state of practical astronomy, it becomes more necessary to develop from the theory (that is from the general properties and interactions of matter) all circumstances that can have an influence on a celestial body: to take advantage from a good observation, as much as it can give.

Although it is true that we can become aware of considerable deviations from a taken rule by observation and by chance: as it was the case with the aberration of light. Yet deviations can exist which are so small, so that it is hard to decide whether they are true deviations or observational errors. Also deviations can exist, which are indeed considerable-but if they are combined with quantities whose determination is not completely finished, they can escape the notice of an experienced observer.

Of the latter kind may also be the deflection of a light ray from the straight line, when it comes near to a celestial body, and therefore considerably experiences its attraction. Since we can easily see that this deflection is greatest when (as seen at the surface of the attracted body) the light ray arrives in horizontal direction, and becomes zero in perpendicular direction, then the magnitude of deflection will be a function of height. However, since also the ray-refraction is a function of height, then these two quantities must be mutually combined: therefore it might be possible, that the deflection would amount several seconds in its maximum, although it couldn't be determined by observations so far.

These are nearly the considerations, which drove me to still think about the perturbation of light rays, which as far as I know was not studied by anyone.

Before I start the investigation, I still want to give some general remarks, by which the calculation will be simplified. Since at the beginning I only want to specify the maximum of such a deflection, I horizontally let pass the light at the location of observation (at the surface of the attracting body), or I assume that the star from which it comes, is apparently rising. For convenience of the study we assume: the light ray doesn't arrive at the place of observation, but emanates from it. We can easily see, that this is completely irrelevant for the determination of the figure of the trajectory. Furthermore if a light ray arrives at a point at the surface of the attracting body in horizontal direction, and then again continues its way (at the beginning horizontally again): then we can easily see, that with this continuation it describes the same curved line, which it has followed until here. If we draw through the place of observation and the center of the attracting body a straight line, then this line will be the major axis of the curved one for the 
trajectory of light; by describing over and under this line two fully congruent sides of the curved line.

$\mathrm{C}$ (Figure 1) shall now be the center of the attracting body, $\mathrm{A}$ is the location at its surface. From A, a light ray goes into the direction $\mathrm{AD}$ or in the horizontal direction, by a velocity with which it traverses the way $\mathrm{v}$ in a second. Yet the light ray, instead of traveling at the straight line $\mathrm{AD}$, will forced by the celestial body to describe a curved line AMQ, whose nature we will investigate. Upon this curved line after the time (calculated from the instant of emanation from $\mathrm{A}$ ), the light ray is located in $\mathrm{M}$, at the distance $\mathrm{CM}=r$ from the center of the attracting body. $g$ be the gravitational acceleration at the surface of the body. Furthermore $\mathrm{CP}=x$, MP $=y$ and the angle $\mathrm{MCP}=h$. The force, by which the light in $\mathrm{M}$ will be attracted by the body into the direction MC, will be $2 g^{-2}$. This force can be decomposed into two other forces,

$$
2 g / r^{2} \cos h \text { and } 2 g / r^{2} \sin h,
$$

into the directions $x$ and $y$, and for that we obtain the following two equations (s. Traité de mécanique céleste par Laplace, Tome I, pag. 21)

$$
\begin{aligned}
\mathrm{dd} x / \mathrm{d} t^{2} & =-(2 g \cos h) / r^{2} \\
\mathrm{dd} y / \mathrm{d} t^{2} & =-(2 g \sin h) / r^{2}
\end{aligned}
$$

If we multiply the first of these equations by $-\sin h$, the second one by $\cos h$, and sum them up, then we obtain:

$$
(\mathrm{dd} y \cos h-\mathrm{dd} x \sin h) / \mathrm{d} t^{2}=0
$$

Now we multiply the first one by $\cos h$, the second one by $\sin h$ and sum them together, then we obtain:

$$
(\mathrm{dd} y \cos h+\mathrm{dd} x \sin h) / \mathrm{d} t^{2}=-2 g / r^{2}
$$

To reduce in these equations the number of variable quantities, we want to express $x$ and $y$ by $r$ and $h$. We easily see that

$$
x=r \cos h ; y=r \sin h
$$

If we differentiate, then we will obtain:

$$
\mathrm{d} x=\cos h \mathrm{~d} r-r \sin h \mathrm{~d} h ; \mathrm{d} y=\sin h \mathrm{~d} r+r \cos h \mathrm{~d} h
$$

And if we differentiate again,

$$
\mathrm{dd} x=\cos h \mathrm{~d} \mathrm{~d} r-2 \sin h \mathrm{~d} h \mathrm{~d} r-r \sin h \mathrm{~d} d h-r \cos h \mathrm{~d} h^{2}
$$

and

$$
\mathrm{dd} y=\sin h \mathrm{~d} d r+2 \cos h \mathrm{~d} h \mathrm{~d} r+r \cos h \mathrm{~d} \mathrm{~d} h-r \sin h \mathrm{~d} h^{2}
$$

If we substitute these values for $\mathrm{dd} x$ and $\mathrm{dd} y$ in the previous equations, the we obtain from (III): $(\mathrm{dd} y \cos h-\mathrm{dd} x \sin h) / \mathrm{d} t^{2}=(2 \mathrm{~d} h \mathrm{~d} r+r \mathrm{dd} h) / \mathrm{d} t^{2}$

Thus we have:

$$
(2 \mathrm{~d} h \mathrm{~d} r+r \mathrm{~d} d h) / \mathrm{d} t^{2}=0
$$

And furthermore by (IV), 


$$
\left(\mathrm{dd} r-r \mathrm{~d} h^{2}\right) / \mathrm{d} t^{2}=-2 g / r^{2}
$$

To make Equation (V) a true differential quantity, we multiply it by $r \mathrm{~d} t$, thus:

$$
\left(2 r \mathrm{~d} h \mathrm{~d} r+r^{2} \mathrm{dd} h\right) / \mathrm{d} t=0
$$

and if we again integrate, we will obtain:

$$
r^{2} \mathrm{~d} h=C \mathrm{~d} t
$$

where $C$ is an arbitrary constant magnitude. To specify $C$, we note that $r^{2} \mathrm{~d} h(=r r \mathrm{~d} h)$ is equal to: the double area of the small triangle which described the radius vector $r$ in the time $\mathrm{d} t$. The double area of the triangle that is described in the first second of time, is however: $=\mathrm{AC} v$, thus we have $C=\mathrm{AC} v$. And if we assume the radius AC of the attracting body as unity, what we will always do in the following, then $C=v$. If we substitute this value for $C$ into the previous equations, then: $r^{2} \mathrm{~d} h=v \mathrm{~d} t$,

Thus we have

$$
\mathrm{d} h=v \mathrm{~d} t / r^{2}
$$

If this value for $\mathrm{d} h$ is substituted into Equations (VI), we obtain:

$$
\left(\mathrm{dd} r / \mathrm{d} t^{2}\right)-v^{2} / r^{3}=-2 g / r^{2}
$$

If we multiply this equations by $2 \mathrm{~d} r$, then:

$$
(2 \mathrm{~d} r \mathrm{dd} r) / \mathrm{d} t^{2}-2 v^{2} \mathrm{~d} r / r^{3}=4 g \mathrm{~d} r / r^{2}
$$

and if we integrate again,

$$
\mathrm{d} r^{2} / \mathrm{d} t^{2}+v^{2} / r^{3}=4 g / r+D
$$

where $D$ is a constant magnitude, that depends on the constant magnitudes which are contained in the equation. From this equation that is found now, the time can be eliminated, hence:

$$
\mathrm{d} t=\mathrm{d} r /\left(D+4 g / r-v^{2} / r^{2}\right)^{1 / 2}
$$

If we substitute this value for $\mathrm{d} t$ into Equation (VII), then we obtain:

$$
\mathrm{d} h=v \mathrm{~d} r / r^{2}\left(D+4 g / r-v^{2} / r^{2}\right)^{1 / 2}
$$

To integrate this equations, we bring it into the form:

$$
\mathrm{d} h=v \mathrm{~d} r / r^{2}\left[D+4 g^{2} / v^{2}-(v / r-2 g / v)^{2}\right]^{1 / 2}
$$

Now we put

$$
v / r-2 g / v=z
$$

then we have $v \mathrm{~d} r / r^{2}=-\mathrm{d} z$

If this and $z$ is substituted into the equation for $\mathrm{d} h$, the we will have:

$$
\mathrm{d} h=-\mathrm{d} z /\left(D+4 g^{2} / v^{2}-z^{2}\right)^{1 / 2}
$$

From that the integral is now: $h=\arccos z /\left(D+4 g^{2} / v^{2}\right)^{1 / 2}+\alpha$ 
where $\alpha$ is a constant magnitude. By well-known properties it is furthermore:

$$
\cos (h-\alpha)=z /\left(D+4 g^{2} / v^{2}\right)^{1 / 2}
$$

and if we also substitute instead of $z$ its value:

$$
\cos (h-\alpha)=\left(v^{2}-2 g r\right) / r\left(v^{2} D+4 g^{2}\right)^{1 / 2}
$$

$h-\alpha$ would be the angle that $r$ forms with the major axis of the curved line that has to be specified. Since furthermore $h$ is the angle which $r$ forms with the line AF (the axis of the coordinates $x$ and $y$ ), then a must be the angle that forms the major axis with the line AF. However, since AF goes through the observation place and the center of the attracting body, then by the preceding, AF must be the major axis; also $\alpha 0$, and thus:

$$
\cos h=\left(v^{2}-2 g r\right) / r\left(v^{2} D+4 g^{2}\right)^{1 / 2}
$$

For $h=0$ it must be $r=\mathrm{AC}=1$, and we obtain from this equation:

$$
\left(v^{2} D+4 g^{2}\right)^{1 / 2}=v^{2}-2 g
$$

If we substitute this in the previous equation, then the still unknown $D$ and also the square-root sign vanish; and we obtain:

$$
\cos h=\left(v^{2}-2 g r\right) / r\left(v^{2}-2 g\right)
$$

furthermore by that

$$
r+\left[\left(v^{2}-2 g\right) / 2 g\right] r \cos h=v^{2} / 2 g
$$

From this finite equation between $r$ and $h$, the curved line can be specified. To achieve this more conveniently, we again want to reduce the equation to coordinates. Let (Figure 1) AP $=x$ and $\mathrm{MP}=y$, then we have:

$$
x=1-r \cos h ; y=r \sin h
$$

and

$$
r=\left[(1-x)^{2}+y^{2}\right]^{1 / 2}
$$

If we substitute this into equation (VIII), then we find:

$$
y^{2}=\left[v^{2}\left(v^{2}-4 g\right) / 4 g^{2}\right](1-x)^{2}-\left[v^{2}\left(v^{2}-2 g\right) / 2 g^{2}\right](1-x)+v^{2} / 4 g^{2}
$$

and if we properly develop everything,

$$
y^{2}=v^{2} x / g+\left[v^{2}\left(v^{2}-4 g\right) / 4 g^{2}\right] x^{2} / 4 g^{2}
$$

Since this equation is of second degree, then the curved line is a conic section, that can be studied more closely now.

If $p$ is the parameter and $a$ the semi-major axis, then (if we calculate the abscissa with its start at the vertex) the general equation for all conic sections is:

$$
y^{2}=p x+p x^{2} / 2 a
$$

This equation contains the properties of the parabola, when the coefficient of $x^{2}$ 
is zero; that of the ellipse when it is negative; and that of the hyperbola when it is positive. The latter is evidently the case in our equation (IX). Since for all our known celestial bodies $4 g$ is smaller than $V^{2}$, then the coefficient of $x^{2}$ must be positive.

If thus a light ray passes a celestial body, then it will be forced by the attraction of the body to describe a hyperbola whose concave side is directed against the attracting body, instead of progressing in a straight direction.

The conditions, under which the light ray would describe another conic section, can now easily be specified. It would describe a parabola when $4 g=v^{2}$, an ellipse when $4 g$ were greater than $v^{2}$, and a circle when $2 g=v^{2}$. Since we don't know any celestial body whose mass is so great that it can generate such an acceleration at its surface, then the light ray always describes a hyperbola in our known world.

Now, it only remains to investigate, to what extend the light ray will be deflected from its straight line; or how great is the perturbation angle (which is the way I want to call it).

Since the figure of the trajectory is now specified, we can consider the light ray again as arriving. And because I at first want to specify only the maximum of the perturbation angle, I assume that the light ray comes from an infinitely great distance. The maximum must take place in this case, because the attracting body longer acts on the light ray when it comes from a greater than from a smaller distance. If the light ray comes from an infinite distance, then its initial direction is that of the asymptote BR (Figure 1) of the hyperbola, because in an infinitely great distant the asymptote falls into the tangent. Yet the light ray comes into the eye of the observer in the direction $\mathrm{DA}$, thus $\mathrm{ADB}$ will be the perturbation angle. If we call this angle $\omega$, then we have, since the triangle $\mathrm{ABD}$ at $\mathrm{A}$ is right-angled:

$$
\operatorname{tang} \varpi=\mathrm{AB} / \mathrm{AD}
$$

However, it is known from the nature of the hyperbola, that $\mathrm{AB}$ is the semi-major axis, and $\mathrm{AD}$ the semi-lateral axis. Thus this magnitudes must also be specified. When $a$ is the semi-major axis, and $b$ the semi-lateral axis, then the parameter is:

$$
p=2 b^{2} / a
$$

If we substitute this value into the general equation of hyperbola

$$
y^{2}=p x+p x^{2} / 2 a
$$

then it transforms into:

$$
y^{2}=2 b^{2} x / a+b^{2} x^{2} / a^{2}
$$

If we compare this coefficients of $x$ and $x^{2}$ with those in (IX), then we obtain the semi-major axis

$$
a=2 g /\left(v^{2}-4 g\right)=\mathrm{AB}
$$

the semi-lateral axis

$$
b=v /\left(v^{2}-4 g\right)^{1 / 2}=\mathrm{AD}
$$


If we substitute this values for $\mathrm{AB}$ and $\mathrm{AD}$ into the expression for $\operatorname{tang} \omega$, then we have:

$$
\tan g \omega=2 g / v\left(v^{2}-4 g\right)^{1 / 2}
$$

We now want to give an application of this formula on earth, and investigate, to what extend a light ray is deflected from its straight line, when it passes by at the surface of earth.

Under the presupposition, that light requires 564.8 seconds of time to come from the sun to earth, we find that it traverses 15.562085 earth radii in a second. Thus $v=15.562085$. If we take under the geographical latitude its square of the sine $1 / 3$ (that corresponds to a latitude of $35^{\circ} 16^{\prime}$ ), the earth radius by $6,369,514$ meters, and the acceleration of gravity by 3.66394 meters (s. Traité de mécanique céleste par Laplace, Tome I, pag. 118): then, expressed in earth radii, $g=$ 0.000000575231 . I use this arrangement, to take the most recent and most reliable specifications of the size of earth's radius and the acceleration of gravity, without specific reduction from the Traité de mécanique céleste. By that, nothing will be changed in the final result, because it is only about the relation of the velocity of light to the velocity of a falling body on earth. The earth radius and the acceleration of gravity must therefore taken under the mentioned degree of latitude, since the earth spheroid (regarding its physical content) is equal to a sphere which has earth's radius (or 6,369,514 meters) as its radius.

If we substitute these values for $v$ and $g$ into the equation of tang $\omega$, then we obtain (in sexagesimal seconds) $\omega=0 " .0009798$, or in even number, $\omega=0 " .001$. Since this maximum is totally insignificant, it would be superfluous to go further; or to specify how this value decreases with the height above the horizon; and by what value it decreases, when the distance of the star from which the light ray comes, is assumed as finite and equal to a certain size. A specification that would bear no difficulty.

If we want to investigate by the given formula, to what extend a light ray is deflected by the moon when it passes the moon and travels to earth, then we must (after the relevant magnitudes are substituted and the radius of the moon is taken as unity) double the value that was found by the formula; because the light ray that passes the moon and falls upon earth, describes two arms of the hyperbola. But nevertheless the maximum must still be much smaller than that of earth; because the mass of the moon, and thus g, is much smaller. The inflexion must therefore only stem from cohesion, scattering of light, and the atmosphere of the moon; the general attraction doesn't contribute anything significant.

If we substitute into the formula for tang $\omega$ the acceleration of gravity on the surface of the sun, and assume the radius of this body as unity, then we find $\omega=$ $0 " .84$. If it were possible to observe the fixed stars very nearly at the sun, then we would have to take this into consideration. However, as it is well known that this doesn't happen, then also the perturbation of the sun shall be neglected. For light rays that come from Venus (which was observed by Vidal only two minutes from the border of the sun, s. Hr. O. L. v. Zachs monatliche Correspondenz etc. II. 
Band pag 87.) it amounts much less; because we cannot assume the distances of Venus and Earth from the sun as infinitely great.

By combination of several bodies, that might be encountered by the light ray on its way, the results would be somewhat greater; but certainly always imperceptible for our observations.

Thus it is proven: that it is not necessary, at least at the current state of practical astronomy, to consider the perturbation of light rays by attracting celestial bodies.

Now I must anticipate two objections, that possibly could raised against me.

One will notice, that I departed from the ordinary method, because I specified several general properties of curved lines before the calculation; which is what usually happens only after, and which might also could have happened at this place. Yet the calculation was very shortened by that, and why should we calculate, when that what has to be proven, can be shown much more evident by a little reasoning?

Hopefully no one finds it problematic, that I treat a light ray almost as a ponderable body. That light rays possess all absolute properties of matter, can be seen at the phenomenon of aberration, which is only possible when light rays are really material. And furthermore, we cannot think of things that exist and act on our senses, without having the properties of matter.

nihil est quod possis dicere ab omni

corpore seiunctum secretumque esse ab inani,

quod quasi tertia sit numero natura reperta.

Lucretius de nat. rer. I, 431

Furthermore I don't think that it is necessary for me to apologize, that I published this investigation; since the result leads to the imperceptibility of all perturbations. Because it also must be even nearly as important for us to know what exists according to the theory, but which has no perceptible influence in practice; as it concerns us, what has a real influence in respect to practice. Our knowledge will be equally extended by both. For example, we prove that the diurnal aberration, the disturbance of the rotation of earth and other such things in addition are imperceptibly small.

\section{Appendix 2}

Example of a calculation of the deflection of the light passing near the sun with the universal gravitation of Newton:

A photon passing near the Sun is submitted to an attractive force (Figure A1). First, we calculate the deviation from the ratio of the speeds in a point such that $\operatorname{tg} \alpha=\Delta v p / c$, with $\Delta v p=$ perpendicular speed to the initial direction and $c=$ speed of the light.

$$
m \cdot \Delta v=F \cdot \Delta t
$$

or

$$
F=m g
$$




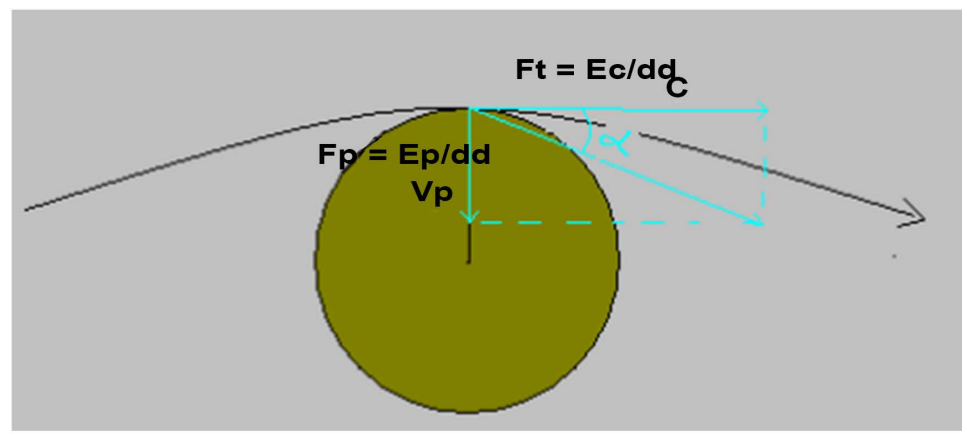

Figure A1. Bending of the light caused by the attractive force.

$$
g=\left(G M / d^{2}\right) \cdot u
$$

with $G$, the gravitational constant, $M$ mass of the sun $1.99 \times 10^{30} \mathrm{~kg}$ and $\mathrm{d}$ the distance between the point where is the photon and the sun, with (1) (2), (a) becomes

$$
\Delta v=\left(G M / d^{2}\right) \cdot \Delta t
$$

$\Delta t$ is the time of the travel of the photon, so $\Delta t=2 d / c$ since $d$ is the distance from the point being in -infinity and the tangential point to the sun and so the distance between the tangential point to the sun and +infinity. Replacing $\Delta t$ by $2 d / c$, so (a') becomes

$$
\Delta v=(2 G M / d c) \cdot u
$$

The angle between $\Delta v$ and $\Delta v p$ is extremely weak (or between the distance $d$ and the perpendicular motion $d d$ ), so we can use the astronomic simplification where the tangent tang a in radians is equal to $1 / d$. So, we have $\Delta v p \sim(1 / d) \Delta v$ and with (a"), we obtain $\Delta v p=\left(2 G M / d^{2} c\right) \cdot u$

(with a more rigorous way, when the photon comes, we have to consider the angle a and multiply by $1 / d$; when the photon goes away, so the angle is $\Pi$-a and we have to multiply by $-1 / d$ )

$$
\operatorname{tang} \alpha=\Delta v p / c=2 G M / d^{2} c^{2}
$$

when a point is between -infinity and the tangential point to the sun;

$\operatorname{tang} \alpha=\Delta v p / c=-2 G M / d^{2} c^{2}$ when a point is between the tangential point to the sun and +infinity)

The total angle of deviation $A$ is calculated by integrating $d$ between -infinity and $R$ and between $R$ and +infinity, $R$ is the radius of the sun

$A \approx \operatorname{tang} A \approx \int\left(2 G M / d^{2} c^{2}\right) d d$ so between -infinity and $R: A^{\prime}=2 G M / R c^{2}$, thus between -infinity and + infinity, $A=4 G M / R c^{2}$. It is this value of $A$ that we find by calculating from the general relativity.

Also, the deviation can be calculated from the forces and from the work of these forces if we consider the work $W p$ of the perpendicular force Fp to the initial direction and the work $\mathrm{Wt}$ of the la tangential force Ft to the initial direction, so $W p=F p d d ; W t=F t d d$. So the deviation $\alpha$ in a point is $\operatorname{tang} \alpha=F p / F t=W p / W t$. Like the tangential speed cannot exceed $c$, the work 
$W t$ always equals the kinetic energy $E c$ corresponding to a speed $c$. The work $W p$ can be calculated from the "potential" energy E. EP is the perpendicular component.

$E=m g d$ with $\mathrm{m}$ mass of the photon, $g$ gravitational acceleration, $d$ distance from the center of the sun to the photon $g=G M / d^{2}$ thus $E=m G M / d$. Like previously, we can write $E p=E(1 / d)$ so $E p=m G M / d^{2} \quad E c=1 / 2\left(m c^{2}\right)$ with $c$, the speed of the photon at $3 \times 10^{8} \mathrm{~m} \cdot \mathrm{s}^{-1}$ (difficult to read for a photon without mass! But, fortunately this mass disappears during the calculation).

The angle of deviation $\alpha$ of the photon in a point of the trajectory is:

$$
\operatorname{tang} \alpha=W p / W t=E p / E c=\left(2 G M / c^{2}\right) \cdot\left(1 / d^{2}\right)
$$

The total deviation for all the trajectory is given by the integral of the Equation (3) as above.

Numerical application:

With a solar radius $R$ of $6958 \times 10^{5} \mathrm{~m}$,

The deviation between -infinity and $R$ is equal to 0.876 ", Similarly the deviation between $R$ and + infinity is equal to $0.876 "$

Thus, the total deviation from -infinity to +infinity is 1.752"; this result is in perfect conformity with the observation and so precise this from the general relativity. 